\title{
Genetic and environmental contributions to depression in Sri Lanka
}

Harriet A. Ball, Athula Sumathipala, Sisira H. Siribaddana, Yulia Kovas, Nick Glozier, Peter McGuffin and Matthew Hotopf

\section{Background}

Susceptibility to depression results from genetic and nonfamilially shared environmental influences in high-income, Western countries. Environments may play a different role for populations in different contexts

\section{Aims}

To examine heritability of depression in the first large, population-based twin study in a low-income country.

\section{Method}

Lifetime depression and a broader measure of depression susceptibility (D-probe) were assessed in 3908 adult twins in Sri Lanka (the CoTASS study).

\section{Results}

There were gender differences for the broad definition

(D-probe), with a higher genetic contribution in females (61\%) than males (4\%). Results were similar for depression, but the prevalence was too low to estimate heritability for males.

\section{Conclusions}

Genetic influences on depression in women appear to be at least as strong in this Sri Lankan sample as in higher-income countries. Conclusions are less clear for men but suggest a larger role for environments rather than genes. The nature as well as the magnitude of environmental influences may also differ across populations.

\section{Declaration of interest}

P.M. has received honoraria from Eli Lilly and GSK and has acted as a consultant in the recent past for GSK and Astra Zeneca. N.G. has received honoraria from Sanofi-Aventis and Servier.
Twin and family studies have estimated the heritability of liability to major depression at $30-50 \%$ when measured in community samples from high-income countries ${ }^{1-4}$ with a somewhat higher estimate in a hospital-based study. ${ }^{5}$ A range of environmental risk factors may contribute to the remaining variance in liability, including adverse life events, poverty, childhood adversity and physical disease. Examining populations where we might expect the nature, magnitude and frequency of environmental risk factors to vary compared with Western countries may shed light on the identity of true environmental causal factors. However, twin studies outside of North America, Europe and Australia are scarce, despite depression being a pressing public health problem in lowand middle-income countries. ${ }^{6}$ If there are large variations in environmental exposures within a population (e.g. access to basic material resources) we might expect depression to have a lower heritability because a greater proportion of variance is explained by these environmental factors. Alternatively, if a very high proportion of the population is exposed to relevant environmental risks, heritability may be higher (see Rutter et al for a review). ${ }^{7}$ It is also important to consider that some apparently 'environmental' risk factors such as stressful life events show gene-environment correlations, with genetic influences on variation in exposure to such risk factors. ${ }^{8,9}$ In this paper we describe the heritability of depression, and gender differences in heritability, in a population-based twin study in Colombo, Sri Lanka (the Colombo Twin And Singleton Study, CoTASS).

\section{Method}

The study received research ethics approval from the Institute of Psychiatry, King's College London, the Ethical Review Committee, University of Sri Jayewardanepura, and the World Health Organization's Research Ethics Committee.

\section{Study design and participants}

This was a population-based twin and non-twin sample, although the current study focuses on the twins only. Full details of the design and implementation of the study are described elsewhere. ${ }^{10}$ The study took place in the Colombo District of Sri Lanka, an area with a population of 2.2 million which includes the island's capital and surrounding areas ( $45 \%$ of the district is classed as rural). ${ }^{11}$ The survey capitalised on the annual update of the electoral register, which consists of a census of all households conducted by local civil servants. We added a question asking whether the householder knew of any twins, and identified 19302 individuals who were twins by this method. From these, 4387 participants were randomly selected and were eligible to take part in the project on common mental disorders, of whom 4024 (91.7\%) actually participated, including 1954 complete pairs of twins. Individuals were excluded if they were under 15 years of age, failed a Mini-Mental State Examination or when interviews were conducted via a proxy.

The mean age of the participants was 34 years, $46 \%$ were male, $53 \%$ were married and $90 \%$ were of Sinhala ethnicity; the employment rate was $73 \%$ for men and $35 \%$ for women; the mean number of people in the participants' households was 4.9. ${ }^{12}$ The median household income for this region of the country in 2006/7 was 24711 Sri Lanka rupees (LKR) per month (approximately 233 US dollars), compared with 16735 LKR for the whole of Sri Lanka. ${ }^{12}$ Sri Lanka has more equitable health and social care than South-East Asian neighbours, ${ }^{13}$ and greater gender equality at least as shown in primary and secondary education, ${ }^{14}$ and its population experiences considerable variation in poverty, rather than there being a very large economic underclass. Despite the relatively high standards of education, approximately a third had received 10 or fewer years of education (note that the adults in the study may have been of school age several decades ago), 
whereas almost a third received 13 or more years. ${ }^{15}$ Life expectancy at birth in Sri Lanka is 69 for men and 76 for women (and 'healthy life expectancy' is 59 for men and 64 for women). ${ }^{16}$

Interviews took place between 2006 and 2007, when Sri Lanka had been experiencing violent civil war for over 20 years. There have been uprisings and bombing attacks in Colombo, and at times a strong military presence. However, this region has not been as heavily involved as have areas in the north and east of the island. Nonetheless, a small minority $(2.6 \%)$ of the participants reported directly participating in the conflict as combatants. Similarly, although many people in Colombo have been affected by the tsunami of 2004, direct involvement was not on the same scale as in the south of the island.

\section{Measures}

Research workers visited the twins' homes to interview them separately. Interviews were administered including the World Health Organization's Composite International Diagnostic Interview. ${ }^{17}$ This gives DSM/ICD diagnoses of mental disorders. For present analyses we used lifetime DSM-IV definitions, ${ }^{18}$ excluding opt-outs because of bereavement or mixed states. We also excluded functional impairment criteria (seeking medical help, or describing the symptoms as interfering with life's activities). This was because of the relatively low likelihood that individuals who met symptom criteria also met functional impairment criteria. ${ }^{15}$ The diagnosis excluding the impairment requirement resulted in a phenotype that was of comparable severity to full DSM depression in Western countries; and within the Sri Lankan sample, the definition excluding an impairment requirement produced an affected group that had very similar symptom characteristics to the affected group according to the full DSM definition. The low reporting of functional impairment may have been as a result of stigma, culturally or economically determined differences in response to depressive symptoms, or the very low proportion of psychiatrists per head of population. Pragmatically, relaxing impairment criteria allowed sufficient power for twin model fitting and there are good precedents for this in recent studies elsewhere (e.g. Kendler et al). ${ }^{2,3}$ The group meeting symptom criteria regardless of impairment is henceforth referred to as the depression group.

In order to assess aspects of depression that may not manifest as full diagnoses, we also examined a broader group who had had at least one 'core' DSM symptom of depression most of the day for nearly every day for a minimum of 2 weeks (sad mood, or loss of interest, as a probable psychiatric symptom rather than because of physical illness or drugs). ${ }^{18}$ This second definition identifies people who are susceptible to depression, and is referred to as the $\mathrm{D}$-probe group. Both the depression and the D-probe definitions refer to lifetime-ever experiences. The sociodemographic risks were comparable between the D-probe and depression definitions, which were also similar to those for a definition that included functional impairment. ${ }^{15}$

Zygosity was assessed using a validated questionnaire ${ }^{19,20}$ administered to both twins.

\section{Analysis}

A database was constructed in SPSS version 14 for Windows. The descriptive statistics were performed in Stata version 9.2 for Windows.

\section{Twin concordance and genetic model fitting}

Classic twin studies examine the similarity of monozygotic (MZ) pairs of twins, and compare this to the similarity of dizygotic (DZ) pairs of twins, in order to tease apart the contributions of three factors to individual differences in a phenotype such as depression. These factors are additive genetics $(A)$, shared environments $(C)$ and non-shared environments $(E)$.

The probandwise concordance rate (PWCR) provides a simple description of similarity within pairs of twins for a binary trait such as presence/absence of depression. The PWCR is calculated as the number of individuals in concordantly affected pairs divided by the total number of affected individuals. Thus it is the probability that the co-twin of a proband will also have the disorder, ${ }^{21}$ and describes cross-twin similarity in a way that can be more intuitive than genetic model fitting. The PWCRs were calculated for each gender $\times$ zygosity group, assuming complete ascertainment of cases.

We used genetic model fitting in $\mathrm{Mx}$ for Windows (www.vcu.edu/mx/index.html) to estimate the relative contributions (and confidence intervals) of $A, C$ and $E$. Greater similarity (i.e. higher correlations) within $\mathrm{MZ}$ than $\mathrm{DZ}$ pairs indicates $A$, since it is assumed that the greater proportion of shared genes is the only explanation for such greater similarity; $C$ includes any environmental exposure that makes twins within a pair (both $\mathrm{MZ}$ and DZ) similar to one another (e.g. family-wide poverty); $E$ reflects any environmental factors that make one twin different from the co-twin (e.g. an accident that affects only one twin within a pair), and so it is calculated as the amount of dissimilarity within MZ pairs. Tetrachoric correlations were used because the data are binary. This method assumes that liability to depression is normally distributed throughout the population, with affected individuals having exceeded a certain threshold of liability.

Age and gender are biologically determined factors that cannot be outcomes of depression, but which may index subgroups of people for whom the nature of the disorder and the contributions of aetiological influences (i.e. $A, C$ and $E$ ) to the disorder differ. In twin models, gender is commonly accounted for by analysing the male and female groups separately, and testing whether the model parameters can be equated across the gender groups. When analysing the D-probe phenotype, we used a five-group model with groups for $\mathrm{MZ}$ male, $\mathrm{MZ}$ female, $\mathrm{DZ}$ male, DZ female and DZ opposite-gender pairs. However, for depression, the number of concordantly affected male pairs was low, so we used a twogroup model (MZ female and DZ female pairs only). The effect of age was accounted for through regression coefficients on the liability thresholds, as has been described previously; ${ }^{22}$ in the current study, these allowed for linear and quadratic effects of age, separately for each gender.

We tested a series of nested models to find the most parsimonious solution. First, we tested for qualitative gender differences, (i.e. whether the identity of the causal genes and environments were the same in men and women), by comparing correlations within same-gender and opposite-gender DZ pairs (because the only factor that is differentially shared is gender). We examined the difference in model fit when: both the genetic correlation and shared environmental correlation for DZ opposite-gender twins are freely estimated; and either the genetic correlation or the shared environmental correlation for DZ opposite-gender twins are fixed to be the same as for DZ same-gender twins ( 0.5 for the genetic correlation, or 1.0 for the shared environmental correlation). Second, we tested whether the magnitude of $A, C$ and $E$ parameters could be equated across gender groups (quantitative gender differences). Finally, we tested whether any of the ACE parameters could be dropped (if they make no statistically significant contribution to the model). As only females were examined for depression, we used the first and second steps for the D-probe definition only. 


\section{Results}

\section{Descriptive statistics}

The demographic characteristics of the sample are described in detail elsewhere, ${ }^{10}$ but briefly, the average age was 34.0 years, and included $46.2 \%$ men, $42.5 \%$ MZ pairs, $29.5 \%$ DZ samegender pairs and $27.3 \%$ DZ opposite-gender pairs. Depression was present in $8.2 \%$ of men and $13.6 \%$ of women; D-probe was present in $13.9 \%$ of men and $17.3 \%$ of women (Table 1). The prevalences varied by age, with the highest prevalences occurring in middle age (e.g. the prevalence of D-probe in ascending age quartiles was $10.0 \%, 16.5 \%, 18.9 \%$ and $17.3 \%, F=10.12, P<0.01$ ).

\section{Aetiology}

Among females, the PWCRs for both depression and D-probe were higher in the $\mathrm{MZ}$ than the $\mathrm{DZ}$ same-gender groups (Table 2 ), indicating genetic influences. However, in males the $M Z$ concordance was no higher than that of the DZ same-gender pairs for either depression phenotype. This suggests that shared environmental $(C)$ rather than genetic influences $(A)$ are driving familial resemblance. Non-shared environmental factors are indicated in both genders since the MZ probandwise concordance is short of 1.0. These findings are also reflected in the polychoric correlations (Table 2), which are the basis of the model fitting.

The low male prevalence and low male concordance for depression was reflected in just four MZ male pairs and four DZ male pairs concordant for depression. The low frequency of concordant-affected males would lead to uncertain conclusions if we were to run a twin model. Therefore we present a full gender-limitation twin model for D-probe, but the twin model for depression includes only women.

\section{Model fit}

The fit of the full genetic model to the data can be performed only in the five-group analysis (D-probe), not the two-group depression analysis, because the latter model is 'just identified'. The five-group model was a good fit to the data (Table 3, $\Delta \chi^{2}=0.120$, d.f. $=1, P=0.729$ ).

The statistical significance of the linear and quadratic regression coefficients of the threshold model age correction were tested. Both regression coefficients were significant $($ at $P<0.05)$ in all models tested (i.e. for males and females in the D-probe model, and for females in the depression model), and so were retained in later models that estimated the $A, C$ and $E$ parameters. In the D-probe model, the regression coefficients could not be equated across gender $(P<0.05$ for both the linear and quadratic components tested separately). However, if both the linear and the quadratic components were equated across gender at once, then there was no significant drop in fit $\left(\Delta \chi^{2}=4.314\right.$, d.f. $=2$, $P=0.116)$. Thus, we fitted genetic models using separate regression coefficients for men and women, but we noted that the age correction is similar across gender.
Table 1 Prevalence of lifetime depression and D-probe

\begin{tabular}{|lccc|} 
& \multicolumn{3}{c}{$n(\%)$} \\
\cline { 2 - 4 } & $\begin{array}{c}\text { Male } \\
(n=1785)\end{array}$ & $\begin{array}{c}\text { Female } \\
(n=2084)\end{array}$ & $\begin{array}{c}\text { Total } \\
(n=3869)\end{array}$ \\
\hline Depression & $147(8.2)$ & $284(13.6)$ & $431(11.1)$ \\
\hline D-probe & $248(13.9)$ & $360(17.3)$ & $608(15.7)$ \\
\hline
\end{tabular}

\section{D-probe}

There was no evidence of qualitative gender differences. However, there were significant quantitative gender differences (equating parameters representing $A, C$ and $E$ resulted in: $\Delta \chi^{2}=9.488$, d.f. $=2, P=0.009$ ). When testing the significance of the $A$ and $C$ parameters in the male and female models separately, the $A E$ model was found to be the best fitting model for females (i.e., dropping $C: P=0.630)$. In the full model, genetic factors influenced $61 \%$ of the liability to depression, with the remainder influenced by non-shared environmental influences (Table 3 ).

In the male model, the $\chi^{2}$-test could not distinguish between the $A E$ (i.e. dropping $C: P=0.263, \Delta$ Akaike's information criterion $(\mathrm{AIC})=-0.749$ ) or $C E$ (i.e. dropping $A: P=0.659$, $\triangle \mathrm{AIC}=-1.805)$ models as a better fit, although the $C E$ model was a marginally better fit as indicated by a lower AIC value; $A$ and $C$ could not both be dropped, indicating significant familial influences on D-probe $(P=0.003)$ (Table 3$)$. This inability to distinguish the source of the familial resemblance was despite having over $80 \%$ power to detect a genetic effect on depression in males, based on the effect size found in females. In addition, the estimates of non-shared environmental influence in the full $A C E$ models had non-overlapping confidence intervals in men and women (men: $72 \%$, CI 52-91\%; women: 36\%, CI 25-50\%).

The age effects on D-probe were slightly greater among men and could not be equated across gender without worsening of fit (linear coefficient $\Delta \chi^{2}=3.969$, d.f. $=1, P=0.046$; quadratic coefficient $\Delta \chi^{2}=4.003$, d.f. $=1, P=0.045$ ).

\section{Depression (female-female pairs only)}

The variance in depression accounted for by genetic and environmental factors closely resembled the results for the broader D-probe definition in females, with $59 \%$ of the variance accounted for by genetic factors, and none accounted for by shared environmental factors (Table 4). The best fitting model was the $A E$ model, since the $C$ parameter could be dropped without worsening of fit; the $C E$ model was a significantly worse fit $(P=0.036)$.

\section{Effect of age correction of thresholds}

If not accounted for, age might mimic the effect of $C$ since it is correlated perfectly within twin pairs. The full ACE models were re-run with the age correction parameters set to zero, to examine

Table 2 Probandwise concordance rates (prior to age adjustment) and tetrachoric correlations (estimated using a separate threshold for males and females, and age corrections to the thresholds) by gender and zygosity group

\begin{tabular}{|c|c|c|c|c|}
\hline \multirow[b]{2}{*}{ Gender-zygosity group (twin pairs, $n$ ) } & \multicolumn{2}{|c|}{ Pairwise concordance rate } & \multicolumn{2}{|c|}{ Correlation, $r(95 \% \mathrm{Cl})$} \\
\hline & Depression & D-probe & Depression & D-probe \\
\hline MZ-MM (360) & 0.163 & 0.242 & $0.27(-0.08$ to 0.57$)$ & $0.25(-0.01$ to 0.48$)$ \\
\hline DZ-MM (261) & 0.182 & 0.257 & $0.28(-0.06$ to 0.57$)$ & 0.29 (0.01 to 0.53$)$ \\
\hline MZ-FF (465) & 0.435 & 0.483 & $0.58(0.41$ to 0.72$)$ & $0.63(0.48$ to 0.75$)$ \\
\hline DZ-FF (307) & 0.253 & 0.294 & 0.29 (0.02 to 0.53$)$ & 0.30 (0.06 to 0.51$)$ \\
\hline DZ-OG (528) & 0.242 & 0.256 & $0.28(0.08$ to 0.47$)$ & $0.17(0.00$ to 0.34$)$ \\
\hline
\end{tabular}




\begin{tabular}{|c|c|c|c|c|c|c|c|}
\hline \multirow[b]{2}{*}{ Model } & \multicolumn{3}{|c|}{ Variance components, $\%(95 \% \mathrm{Cl})(n=1921$ pairs $)$} & \multicolumn{4}{|c|}{ Fit indices (compared with full model) } \\
\hline & $\begin{array}{c}\text { A } \\
\text { additive genetic }\end{array}$ & $\frac{C}{\text { shared environment }}$ & $\begin{array}{c}E \\
\text { non-shared environment }\end{array}$ & $\Delta \chi^{2}$ & $\Delta$ d.f. & $P$ & AIC \\
\hline Men (full model) & $4(0-45)$ & $24(0-44)$ & $72(52-91)$ & - & - & - & - \\
\hline Men (AE model) & $28(8-48)$ & [0] & $72(52-92)$ & 1.251 & 1 & 0.263 & -0.749 \\
\hline Men (CE model) & [0] & $27(10-44)$ & $73(56-90)$ & 0.195 & 1 & 0.659 & -1.805 \\
\hline Men (E model) & [0] & [0] & 100 & 11.546 & 2 & 0.003 & 7.546 \\
\hline Women (Full model) & $61(19-75)$ & $3(0-39)$ & $36(25-50)$ & - & - & - & - \\
\hline Women (AE model) & $64(50-76)$ & [0] & $36(24-50)$ & 0.232 & 1 & 0.630 & -1.768 \\
\hline Women (CE model) & [0] & $52(39-63)$ & $48(37-61)$ & 7.907 & 1 & 0.005 & 5.907 \\
\hline Women (E model) & [0] & [0] & 100 & 63.174 & 2 & $<0.001$ & 59.174 \\
\hline
\end{tabular}

\begin{tabular}{|c|c|c|c|c|c|c|c|}
\hline \multirow[b]{2}{*}{ Model } & \multicolumn{3}{|c|}{ Variance components, $\%(95 \% \mathrm{Cl})(n=772$ pairs $)$} & \multicolumn{4}{|c|}{ Fit indices (compared to full model) } \\
\hline & $\begin{array}{c}A \\
\text { additive genetic }\end{array}$ & $\begin{array}{c}C \\
\text { shared environment }\end{array}$ & $\frac{E}{\text { non-shared environment }}$ & $\Delta \chi^{2}$ & $\Delta$ d.f. & $P$ & AIC \\
\hline Women (full model) & $59(4-72)$ & $0(0-48)$ & $41(28-57)$ & - & - & - & - \\
\hline Women (AE model) & $59(43-72)$ & [0] & $41(28-57)$ & 0.00 & 1 & 1.00 & -2.000 \\
\hline Women (CE model) & [0] & $49(35-61)$ & $51(39-65)$ & 4.419 & 1 & 0.036 & 2.419 \\
\hline Women (E model) & [0] & [0] & 100 & 44.497 & 2 & $<0.001$ & 40.497 \\
\hline
\end{tabular}

the amount of error avoided in the parameter estimates by including an age correction. The percentage of the variance accounted for by the $C$ parameter was estimated at 25,3 and 0 , and the proportion of the variance accounted for by the $A$ factor was 6,62 and 61 respectively in the male and female models for D-probe, and the female model for depression. Therefore, although not accounting for age generally led to incorrectly inflated $C$ and heritability estimates, the change in magnitude was very small $(0-2 \%$ of the total variance in liability).

\section{Discussion}

This is the first population-based twin study in a low-income country to assess the heritability of depressive disorder. We identified a true population sample of twins and achieved an exceptionally high participation rate $(>90 \%)$, making our findings likely to be highly generalisable. We took special care to translate the study interview to take account of cultural meanings of depression and related concepts. Despite a large sample size, the relatively low lifetime prevalence of major depression means that we had limited statistical power particularly in men.

We have found that the aetiology of depression in this Sri Lankan context (in terms of the relative contribution of genes and environments) may differ in some respects compared with previous reports from higher-income, Western countries. The heritabilities of liability to depression and to a broader category, D-probe (two weeks of depressed mood or loss of interest), were similar to international estimates among women. The heritability of D-probe was significantly lower (and so non-shared environments contributed more) for men compared with women within this population. This heritability among Sri Lankan men appears considerably lower than in most studies from Europe, Australia and North America; however, this estimate for heritability among men is imprecise and the confidence intervals do still fall within the bounds of estimates shown in European-ancestry populations. Gender differences in depression heritability have inconsistently been reported in other countries, ${ }^{1}$ and where found these have been in the same direction but of smaller magnitude than the present study. ${ }^{2,3}$ However, a gender difference in the same direction as the present study was found in a recent investigation of depressive symptoms in a South Korean volunteer twin sample. ${ }^{23}$

Comparisons of aetiology cross-nationally rely on the detected phenotypes being sufficiently similar. Impacts of confounding factors that may differ cross-nationally should also be considered. This sample had a fairly young age distribution (mean age of 34 years). Differences may have arisen through the way in which participants interpreted psychiatric questions, and their willingness to report symptoms in the face of stigma and cultural interpretations of symptoms of distress. ${ }^{24,25}$ Countering these concerns, the episodes of depression we found ${ }^{15}$ consisted of a similar pattern of particular symptoms, total number of symptoms and sociodemographic correlates as has been found in other countries using full DSM-IV diagnostic criteria. ${ }^{26,27}$ This suggests that the same underlying phenotype has been tapped into, rather than a subtype of depression that is substantially qualitatively different from that seen in other countries.

\section{What can we infer from cross-national heritability comparisons?}

Population genetic evidence suggests that most worldwide genetic variance in humans is seen within populations and only a small percentage of variance exists between populations or between countries. ${ }^{28}$ This implies that much of the genetic influence contributing to heritability estimates will be similar across countries. Therefore it is likely that any heritability differences we see across countries are because of differences in environments, or the extent to which genes co-act and interact with these. Although use of Western definitions of illness may be problematic in non-Western settings, ${ }^{29}$ there is remarkable similarity in symptom and environmental risk-factor profiles between populations. ${ }^{30,31}$ However, the frequency of risk factors such as poverty 
and low educational status may differ between low- and middleincome and high-income countries. Comparing the aetiology results uncovered in the current study with results from a range of higher-income and non-Asian countries is thus likely to be informing us mostly about environmental rather than genetic differences across countries.

The current results are not necessarily generalisable to the whole island of Sri Lanka, in particular because we only sampled in one district that includes the capital city and neighbouring areas, and because the sample was predominantly of Sinhala ethnicity. Nonetheless, we gained a participation rate over $90 \%$ and this is the first large population-based twin study with a mental health focus in a non-Western country. This is important because heritability is a population-specific statistic: we may learn much about the fundamental aetiology of disorders such as depression by examining the relative influence of genes and environments in substantially different contexts.

We found that women's genetic liability to depression in Sri Lanka was if anything slightly higher (although with wide confidence intervals) than that reported in community samples in Western high-income countries. This suggests that the aetiology of female depression is similar across countries of different income levels, countering the hypothesis that greater variation in environmental exposures in Sri Lanka would produce a low heritability of depression. An alternative interpretation is that environmental factors with high variance in Sri Lanka (such as relative poverty) may not be having a large causal effect on female depression. This suggests that the environments that do contribute to female depression comprise factors that have a similar variance (or even a lower variance) in Sri Lanka compared with the West. It is also possible that qualitatively different environmental factors play a role in different countries, and operate through different mechanisms. The Sri Lankan cultural context may exert a blanket effect on women (i.e. an effect that influences most women, therefore with low variance) that emphasises the genetic component of depression, as compared with the Western world. As a speculative example, fewer opportunities for female emancipation in the Sri Lankan context may mean that many women are stuck in unhappy situations. However, those women who perform well at school (partly because of genetically inherited ability) may have a greater influence on the home situation they end up in later in life, thereby influencing their exposure to depressogenic situations. Consequently, a pervasive environmental exposure would not be identified as a shared environmental factor when examining one population using the twin design (because there is no variation in exposure in the population), but could act to increase heritability as measured within that population.

The lower prevalence of depressive disorders in men reduces the power to distinguish between genetic and environmental components of familial similarity, although depression in men was found to be significantly less heritable than in women. In addition, non-shared environmental influences were greater in men than in women. One explanation of the lower male heritability (and greater $E$ ) is a lower reliability of depression reporting among men in Sri Lanka, mimicking greater environmental variation, although there is inconclusive evidence for gender differences in reliability of depression reporting. ${ }^{32,33}$ There appeared to be a slightly greater effect of age on the prevalence of depression in men than in women, which might reflect lower willingness to report depression, or greater salience of certain aetiological risk factors among men of a particular age. The symptom profiles of depressive phenotypes in this sample appear similar across gender, ${ }^{15}$ suggesting that the same disorder is being detected among men and women. Thus the episodes detected are likely to be equally reliable in both genders, although it is still possible for low reliability to exist as one gender is less likely to report any of their symptoms.

On the other hand, it is possible that certain 'real' environmental factors play an important role for the development of depression in men only. For example, certain aspects of standards of living, employment patterns and residence in a more urbanised area appear to be more strongly associated with depression in males than in females in this sample. ${ }^{15}$

\section{Limitations}

All classic twin studies, including the current study, are based on the equal environments assumption, i.e. that the degree of environmental similarity is constant across zygosities. This assumption has been tested and supported in relation to its impact on affective disorders, including depression. ${ }^{34,35}$

The low prevalence of the phenotypes in men led to wide confidence intervals in the genetic model. The upper end of the interval for the male D-probe model does in fact overlap with findings from higher-income, Western countries, but the pattern nonetheless is most consistent with a low heritability in men. One of the most striking findings is the magnitude of the gender difference in the genetic models, which has not been seen in higher-income countries.

In the current study our assessment of depression did not include a requirement for functional impairment, and the D-probe phenotype only required one of two core symptoms of a depressive episode. A low prevalence of depressive episodes that met full DSM criteria reduced power to run genetic models. However, the less stringent phenotypes that we identified do appear to be on the same diagnostic continuum as the full diagnosis (in terms of having similar symptom profiles and sociodemographic associations). Also, the requirement for functional impairment may not be as relevant in this population as for those for which it was designed. ${ }^{15}$ The liability threshold model of disorders assumes that everyone in a population is to a greater or lesser degree liable to develop a disorder, with diagnostic thresholds identifying those above a certain level of liability. Under this assumption, the current study is likely to have tapped into the same dimension as if we had been able to fully analyse a more stringent definition of depression.

In conclusion, these results from a large twin sample in a low/middle-income country suggest that different aetiological processes may be at work in this Sri Lankan setting relative to previously studied countries. As well as highlighting processes particular to Sri Lanka, these differences can inform our general understanding of the aetiology of depression throughout the world.

\footnotetext{
Harriet A. Ball, PhD, MRC Social Genetic and Developmental Psychiatry Centre, Institute of Psychiatry, King's College London, UK; Athula Sumathipala, MBBS, DFM MD, MRCPsych, PhD, Sri Lanka Twin Registry, Institute of Research and Development, Battaramulla, Sri Lanka and Section of Epidemiology, Institute of Psychiatry, King's College London, UK; Sisira H. Siribaddana, MBBS MD, Sri Lanka Twin Registry, Institute of Research and Development, Battaramulla, Sri Lanka; Yulia Kovas, PhD, MRC Social Genetic and Developmental Psychiatry Centre, Institute of Psychiatry, King's College London, UK; Nick Glozier, MSC, PhD, MRCPsych, Sydney Medical School, University of Sydney, Australia, and Department of Psychological Medicine, Institute of Psychiatry, King's College London, UK; Peter McGuffin, MB, PhD, FRCP, Institute of Psychiatry, King's College London, UK; Peter McGuffin, MB, PhD, FRCP,
FRCPsych, FMedSci, MRC Social Genetic and Developmental Psychiatry Centre, FRCPsych, FMedSci, MRC Social Genetic and Developmental Psychiatry Centre,
Institute of Psychiatry, King's College London, UK; Matthew Hotopf, BSc MBBS MSC PhD MRCPsych, Department of Psychological Medicine, Institute of Psychiatry, King's college London, UK
}

Correspondence: Harriet Ball, MRC Social Genetic and Developmental Psychiatry Centre, Institute of Psychiatry, King's College London, De Crespigny Park, London SE5 8AF, UK. Email: harriet.ball@kcl.ac.uk

First received 8 Jan 2009, final revision 29 May 2009, accepted 22 Jul 2009 


\section{Funding}

The Wellcome Trust provided funding for the CoTASS study (grant no. 069629/Z/02/A), and the Institute for Research and Development, Sri Lanka, provided infrastructural support. H.B. was supported by an ESRC research studentship. M.H. is funded by the South London and Maudsley NHS Foundation Trust and Institute of Psychiatry, King's College London, National Institute of Health Research, Biomedical Research Centre.

\section{References}

1 Sullivan PF, Neale MC, Kendler KS. Genetic epidemiology of major depression: review and Meta-analysis. Am J Psychiatry 2000; 157: 1552-62.

2 Kendler KS, Gardner CO, Neale MC, Prescott CA. Genetic risk factors for major depression in men and women: similar or different heritabilities and same or partly distinct genes? Psychol Med 2001; 31: 605-16.

3 Kendler KS, Gatz M, Gardner CO, Pedersen NL. A Swedish national twin study of lifetime major depression. Am J Psychiatry 2006; 163: 109-14.

4 Middeldorp CM, Birley AJ, Cath D, Gillespie NA, Willemsen G, Statham DJ, et al. Familial clustering of major depression and anxiety disorders in Australian and Dutch twins and siblings. Twin Res Hum Genet 2005; 8: 609-15.

5 McGuffin P, Katz R, Watkins S, Rutherford J. A hospital-based register of the heritability of DSM-IV unipolar depression. Arch Gen Psychiatry 1996; 53 : 129-36.

6 Prince M, Patel V, Saxena S, Maj M, Maselko J, Phillips MR, et al. No health without mental health. Lancet 2007; 370: 859-77.

7 Rutter M, Moffitt TE, Caspi A. Gene-environment interplay and psychopathology: multiple varieties but real effects. I Child Psychol Psychiatry 2006; 47: 226-61.

8 Kendler KS, Karkowski-Shuman L. Stressful life events and genetic liability to major depression: genetic control of exposure to the environment? Psychol Med 1997; 27: 539-47.

9 Thapar A, Harold G, McGuffin P. Life events and depressive symptoms in childhood - shared genes or shared adversity? A research note. J Child Psychol Psychiatry 1998; 39: 1153-8.

10 Siribaddana S, Ball H, Hewage S, Glozier N, Kovas $\mathrm{Y}$, Dayaratne DARK, et al. Colombo Twin and Singleton Study (CoTASS). A description of a population based twin study of mental disorders in Sri Lanka. BMC Psychiatry 2008; 8: 49 .

11 Department of Census and Statistics - Sri Lanka. Population and Housing Statistics. Department of Census and Statistics - Sri Lanka, 2001 (http:// www.statistics.gov.lk/PopHouSat/index.asp).

12 Department of Census and Statistics - Sri Lanka. Household Income and Expenditure Survey - 2006/07. Department of Census and Statistics - Sri Lanka, 2008 (http://www.statistics.gov.Ik/HIES/HIES2006_07Website/ Publications/HIES200607Final\%20ReportWeb\%20.pdf).

13 Jayasekara RS, Schultz T. Health status, trends, and issues in Sri Lanka. Nurs Health Sci 2007; 9: 228-33.

14 Asia-Pacific MDG Study Series. The Millennium Development Goals: Progress in Asia and the Pacific 2007. United Nations Development Programme (http://www.undprcc.Ik/Publications/MDGI/MDGI_Progress_In_Asia_and_ the_Pacific_2007.pdf).

15 Ball HA, Siribaddana SH, Kovas Y, Glozier N, McGuffin P, Sumathipala A, et al Epidemiology and symptomatology of depression in Sri Lanka. A crosssectional population-based survey in Colombo District. J Affect Disord 2009; in press: doi 10.1016/j.jad.2009.08.014.
16 World Health Organization. World Health Statistics. WHO, 2008 (http://www. who.int/country/lka/en/).

17 World Health Organization. Composite International Diagnostic Interview (CIDI). WHO, Division of Mental Health, 1990.

18 American Psychiatric Association. Diagnostic and Statistical Manual of Mental Disorders (4th edn, revised) (DSM-IV-TR). APA, 2000.

19 Ooki S, Yamuda K, Asaka A, Hayakawa K. Zygosity diagnosis of twins by questionnaire. Acta Genet Med Gemellol (Roma) 1990; 39: 109-15.

20 Sumathipala A, De Silva N, Siribaddana SH, Abeysingha MR, Fernando DJ. Cross-cultural adaptation and preliminary validation of a zygosity determination questionnaire for twins in Sri Lanka. Twin Res Hum Genet 2000; 3: 205-12.

21 Rijsdijk FV, Sham PC. Analytic approaches to twin data using structural equation models. Brief Bioinform 2002; 3: 119-33.

22 Reynolds CA, Fiske A, Fratiglioni L, Pedersen NL, Gatz M. Heritability of an age-dependent categorical phenotype: cognitive dysfunction. Twin Res Hum Genet 2006; 9: 17-23.

23 Hur YM. Sex differences in genetic and environmental contributions to depression symptoms in South Korean adolescent and young adult twins. Twin Res Hum Genet 2008; 11: 306-13.

24 Goldney RD, Fisher L, Hawthorne G. Depression and the CIDI. Br J Psychiatry 2004; 185: 518-9.

25 Hsu E, Davies CA, Hansen DJ. Understanding mental health needs of Southeast Asian refugees. Historical, cultural, and contextual challenges. Clin Psych Rev 2004; 24: 193-213.

26 Kornstein SG, Schatzberg AF, Thase ME, Yonkers KA, McCullough JP, Keitner $\mathrm{Gl}$, et al. Gender differences in chronic major and double depression. J Affect Disord 2000; 60: 1-11.

27 Middeldorp CM, Wray NR, Andrews G, Martin NG, Boomsma DI. Sex differences in symptoms of depression in unrelated individuals and oppositesex twin and sibling pairs. Twin Res Hum Genet 2006; 9: 632-6.

28 Goldstein DB, Chikhi L. Human migrations and population structure: what we know and why it matters. Annu Rev Genomics Hum Genet 2002; 3: 129-52.

29 Summerfield D. Depression: epidemic or pseudo-epidemic? J $R$ Soc Med 2006; 99: 161-2.

30 Patel V, Araya R, de Lima M, Ludermir A, Todd C. Women, poverty and common mental disorders in four restructuring societies. Soc Sci Med 1999; 49: 1461-71.

31 Simon GE, Goldberg DP, Von KM, Ustun TB. Understanding cross-national differences in depression prevalence. Psychol Med 2002; 32: 585-94.

32 Foley DL, Neale MC, Kendler KS. Reliability of a lifetime history of major depression: implications for heritability and co-morbidity. Psychol Med 1998; 28: $857-70$.

33 Wells JE, Horwood $\mathrm{L}$. How accurate is recall of key symptoms of depression? A comparison of recall and longitudinal reports. Psychol Med 2004; 34: 1001-11.

34 Kendler KS, Neale MC, Kessler RC, Heath AC, Eaves LJ. A test of the equal-environment assumption in twin studies of psychiatric illness. Behav Genet 1993; 23: 21-7.

35 Kendler KS, Prescott CA. A population-based twin study of lifetime major depression in men and women. Arch Gen Psychiatry 1999; 56: 39-44. 